\title{
ARTICLE
}

Acute myeloid leukemia

\section{A proof of concept phase I/II pilot trial of LSD1 inhibition by tranylcypromine combined with ATRA in refractory/relapsed AML patients not eligible for intensive therapy}

\author{
Maxi Wass ${ }^{1} \cdot$ Stefanie Göllner ${ }^{2} \cdot$ Birgit Besenbeck ${ }^{2} \cdot$ Richard F. Schlenk $^{2} \cdot$ Petra Mundmann $^{3}$ - Joachim R. Göthert ${ }^{4}$. \\ Richard Noppeney ${ }^{4}$ Christoph Schliemann ${ }^{5} \cdot$ Jan-Henrik Mikesch ${ }^{5} \cdot$ Georg Lenz ${ }^{5} \cdot$ Martin Dugas $\mathbb{1}^{5}$. \\ Martin Wermke ${ }^{6}$ Christoph Röllig $\mathbb{D}^{6} \cdot$ Martin Bornhäuser $^{6} \cdot$ Hubert Serve $^{7} \cdot$ Uwe Platzbecker $^{8}$.

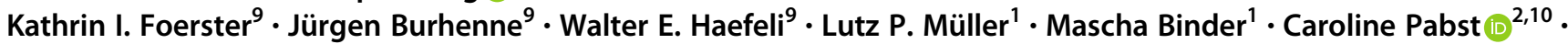 \\ Carsten Müller-Tidow $\mathbb{i D}^{2,10} \cdot$ on behalf of the Study Alliance Leukemia (SAL)
}

Received: 29 August 2019 / Revised: 16 April 2020 / Accepted: 26 May 2020 / Published online: 19 June 2020

(c) The Author(s), under exclusive licence to Springer Nature Limited 2020

\begin{abstract}
All-trans-retinoic acid (ATRA) is highly active in acute promyelocytic leukemia but not in other types of acute myeloid leukemia (AML). Previously, we showed that ATRA in combination with Lysine-specific demethylase 1 (LSD1) inhibition by tranylcypromine (TCP) can induce myeloid differentiation in AML blasts. This phase I/II clinical trial investigated the safety and efficacy of TCP/ATRA treatment as salvage therapy for relapsed/refractory (r/r) AML. The combination was evaluated in 18 patients, ineligible for intensive treatment. The overall response rate was $20 \%$, including two complete remissions without hematological recovery and one partial response. We also observed myeloid differentiation upon TCP/ ATRA treatment in patients who did not reach clinical remission. Median overall survival (OS) was 3.3 months, and oneyear OS 22\%. One patient developed an ATRA-induced differentiation syndrome. The most frequently reported adverse events were vertigo and hypotension. TCP plasma levels correlated with intracellular TCP concentration. Increased $\mathrm{H} 3 \mathrm{~K} 4 \mathrm{me} 1$ and H3k4me2 levels were observed in AML blasts and white blood cells from some TCP/ATRA treated patients. Combined TCP/ATRA treatment can induce differentiation of AML blasts and lead to clinical response in heavily pretreated patients with $\mathrm{r} / \mathrm{r}$ AML with acceptable toxicity. These findings emphasize the potential of LSD1 inhibition combined with ATRA for AML treatment.
\end{abstract}

\section{Introduction}

Epigenetic alterations are a hallmark of acute myeloid leukemia (AML). Targeting epigenetic regulators constitutes a promising approach in leukemia treatment [1]. AML blasts exhibit a profound disruption of epigenetic events, i.e., of the $\mathrm{CpG}$ island methylation status of promoters of a

These authors contributed equally: Caroline Pabst and Carsten Müller-Tidow

Supplementary information The online version of this article (https:// doi.org/10.1038/s41375-020-0892-z) contains supplementary material, which is available to authorized users.

Maxi Wass

maxi.wass@uk-halle.de

Extended author information available on the last page of the article growing number of genes leading to an aberrant silencing of these genes. Histone methylation can be activating $\left(\mathrm{H} 3 \mathrm{~K} 4{ }^{\mathrm{me} 2}\right)$ or inactivating $\left(\mathrm{H} 3 \mathrm{~K} 27^{\mathrm{me} 2}\right)$. The removal of $\mathrm{H} 3 \mathrm{~K} 4^{\mathrm{me} 2}$ is catalyzed by the histone H3 lysine 4 demethylase LSD1, which is highly expressed in blasts from AML patients [2]. Different inhibitors of LSD1 are known, for example tranylcypromine (TCP, trans-2-phenylcyclopropylamine), an irreversible monoamine oxidase (MAO) inhibitor, which has been used as an antidepressant and anxiolytic agent since the 1960s [3]. Recently, we have shown that combinatorial use of all-trans-retinoic acid (ATRA) and inhibitors of LSD1 induced differentiation in non-acute promyelocytic leukemia (APL) AML cell lines and primary patient samples [4]. Upon LSD1 inhibition in HL-60 AML and TEX cells, which mimic the features of primary human AML cells, by TCP, differentiation was significantly increased [4]. In addition, TCP combined with ATRA-induced myelomonocytic differentiation of primary 
AML patient samples and diminished leukemic engraftment of primary AML blasts in a NOD.SCID xenograft mouse model [4]. These data established a crosstalk between the ATRA-induced differentiation pathway and LSD1 inhibition as a targetable pathway in non-APL AML patients and formed the rationale for this clinical trial.

Here, we report the results of a phase I/II study of TCP and ATRA in refractory or relapsed AML patients not considered eligible for intensive salvage chemotherapy.

\section{Patients and methods}

\section{Study design}

The TCP/ATRA trial is a prospective phase I/II open-label, single-arm trial to evaluate safety and efficacy of ATRA combined with TCP in refractory or relapsed patients not eligible for intensive treatment. The trial was conducted at four centers in Germany (Essen, Dresden, Halle, Münster) on behalf of the Study Alliance Leukemia (SAL). Sponsor of the trial was the Martin Luther University Halle-Wittenberg. The trial was approved by the Ethics Committee of the Martin Luther University Halle-Wittenberg and was conducted according to the Declaration of Helsinki (http://clinicaltrials. gov identifier NCT02261779; EudraCT 2012-002154-23).

\section{Patients}

Patients were eligible if aged $\geq 18$ years, with confirmed diagnosis of AML (except AML M3, APL) with relapsed or refractory disease after at least one therapy regime and not considered as fit to receive intensive treatment. Major exclusion criteria were an option of intensive chemotherapy and/or hematopoietic stem cell transplantation (HSCT), an Eastern Cooperative Oncology Group performance status (ECOG PS) $\geq 3$ or a life expectancy $<4$ weeks, uncontrolled hypertension (systolic blood pressure $\geq 140 \mathrm{mmHg}$ ), prolonged corrected QT interval (QTc $>480 \mathrm{~ms}$ ), inadequate organ function, including left ventricular ejection fraction $<40 \%$, renal insufficiency (creatinine clearance $<30 \mathrm{ml} / \mathrm{min}$ ) or hepatic impairment (AST/ALT $>2.5$-fold or Bilirubin $>$ 1.5-fold the upper normal range). Patients with hypersensitivity to Tretinoin, retinoids, vitamin A, soya, peanuts or TCP or with known epilepsy or psychiatric affections as well as patients taking medication with known interaction with Tretinoin or TCP were excluded. All patients gave written informed consent.

\section{Treatment}

All patients received TCP $10 \mathrm{mg}$ orally once daily starting on day one. The dose was increased by $10 \mathrm{mg} /$ day each day up to a maximum dose of $60 \mathrm{mg} /$ day in 7-10 days, depending on patient's tolerability. ATRA was started on day 7 and administered orally at a total dose of $45 \mathrm{mg} / \mathrm{m}^{2}$ per day divided in two doses. In case the TCP dose per day was lower than $50 \mathrm{mg} /$ day, the start of ATRA could be delayed until a TCP dose of $50 \mathrm{mg} /$ day was reached. Before and while on study, treatment with hydroxyurea was allowed to reduce the number of leukocytes. Patients with $>$ 10.000 leukocytes/ $\mu$ l or rapidly rising blast counts could receive dexamethasone if the investigator suspected a high risk of differentiation syndrome. During TCP treatment patients had to follow a low tyramine diet to prevent hypertensive crisis.

At the end of every cycle (day 28), bone marrow analyses were performed for response evaluation. Cycles were scheduled every 4 weeks until disease progression (PD), completed scheduled treatment of 12 months, intolerance/ toxicity or discontinuation upon patient or investigator request.

\section{Study endpoints}

The primary endpoints of the study were safety and response. Patients were followed until death or regular end of study (defined as 12 months after last treatment). Adverse events (AE) were recorded and graded using the National Cancer Institute Common Toxicity Criteria (NCI CTC) version 4.0. The cumulative response rate (complete remission [CR], $\mathrm{CRi}[\mathrm{CR}$ with incomplete recovery of neutrophils or platelets], partial response [PR]) was assessed according to the Cheson response criteria [5]. Stable disease (SD) was defined as reduction by $\leq 50 \%$ of blast counts in peripheral blood and bone marrow versus baseline, or stable percentage of blasts in peripheral blood or bone marrow.

\section{Determinations of tranylcypromine plasma concentrations}

Plasma levels of TCP were analyzed by a central laboratory (Medizinisches Versorgungszentrum Dr. Eberhard \& Partner, Dortmund, Germany) to determine plasma concentrations and association with response.

\section{Liquid chromatography-tandem mass spectrometry for the detection of intracellular tranylcypromine concentrations}

Intracellular TCP concentrations were analyzed using ultrahigh-performance liquid chromatography-tandem mass spectrometry (Waters ACQUITY UPLC Xevo TQ-XS, Eschborn, Germany). TCP concentrations were calculated assuming an average AML cell diameter of $15 \mu \mathrm{m}$. Because TCP irreversibly binds to its target, the assay only 
quantified unbound tranylcypromine (i.e., TCP that is not covalently bound to its target). The measured intracellular TCP concentrations thus reflect a minimum concentration reached in these cells.

TCP hydrochloride was obtained from Sigma-Aldrich (Steinheim, Germany). ${ }^{2} \mathrm{H}_{5}$-TCP (internal standard) was purchased from Santa Cruz Biotechnology (Heidelberg, Germany; purity: 98\%). The UPLC-MS/MS eluent was composed of acetonitrile (Biosolve, Valkenswaard, The Netherlands) and water (arium mini Lab Water Systems, Sartorius, Göttingen, Germany), which were acidified (0.1 vol-\%) with pure formic acid (Sigma-Aldrich, Steinheim, Germany). For sample preparation procedures, boric acid (Merck, Darmstadt, Germany) was dissolved in water to create a borate buffer of $0.2 \mathrm{M}$. Buffer was adjusted to $\mathrm{pH}$ 9.0 with sodium hydroxide (Merck, Darmstadt, Germany). Tert-butyl methyl ether was purchased from Merck (Darmstadt, Germany). Stock solutions used for calibration, internal quality control (QC), and internal standard were prepared in acetonitrile/water (50/50 vol-\%).

To ensure assay stability by balancing losses during sample preparation an internal standard $\left({ }^{2} \mathrm{H}_{5}\right.$-TCP) was added to each cell pellet $\left(1 \times 10^{7}\right.$ cells). Subsequently, borate buffer at $\mathrm{pH} 9.0$ was added to each sample to allow for a liquid-liquid extraction using tert-butyl methyl ether. Chromatography was performed on a reversed-phase column (Synergi $2.5 \mu \mathrm{m}$ Polar-RP $100 \times 2 \mathrm{~mm}$ column; Phenomenex, Aschaffenburg, Germany) in isocratic mode using an eluent mixture of acetonitrile/water with $0.1 \%$ formic acid (60/40 vol-\%). After positive electrospray ionization, mass-to-charge transitions of $\mathrm{m} / \mathrm{z} 134>117$ (TCP) and $\mathrm{m} / \mathrm{z} 139>122\left({ }^{2} \mathrm{H}_{5}\right.$-TCP) were monitored for TCP quantification. The calibration range was linear from $0.1-2000 \mathrm{pg} / 1 \times 10^{7}$ cells. Matrix effects caused a slight ion enhancement $(115 \%)$, which was compensated by the internal standard. The sample preparation ensured that $78 \%$ of TCP was recovered from the sample. Samples were analyzed with within-batch and between-batch accuracy of 94-108\% and precision of $\leq 12 \%$.

\section{Western blot}

Cells were lysed in SDS lysis buffer $(0,1 \%$ SDS, $50 \mathrm{mM}$ Tris $\mathrm{pH}$ 8.0, 10U/ml Benzonase and Protease Inhibitor Cocktail (Sigma-Aldrich, Darmstadt Germany)) for $30 \mathrm{~min}$ at $4{ }^{\circ} \mathrm{C}$. For target detection $0.5 \mu \mathrm{g}$ total protein (for $\mathrm{H} 3 \mathrm{~K} 4 \mathrm{me} 2$ and total $\mathrm{H} 3$ detection) and $5 \mu \mathrm{g}$ total protein (for actin and LSD1 detection) were resolved on $12 \%$ Bis-Tris gels (Invitrogen) by SDS-PAGE, blotted onto nitrocellulose membranes, and probed by standard methods using the following antibodies: anti- $\beta$-actin (Sigma-Aldrich, A5441, 1:5000), anti-total H3 (Abcam, ab39763, 1:5000), antiH3K4me2 (Abcam, ab32356, 1:5000), anti-LSD1 (Cell
Signaling, \# 2139S, 1:1000). Each primary antibody had been validated for the relevant species and applications by the manufacturer.

\section{Statistical analysis}

Baseline characteristics and demographic data of all patients were summarized descriptively, using medians, means and ranges. All patients who received at least one dose of study treatment were analyzed for safety and tolerability, and of those who had at least 14 days of TCP/ATRA treatment also for efficacy. Survival data were evaluated by means of Kaplan-Meier plots. TCP levels between non-responders and responders were analyzed by paired t-test.

\section{Results}

\section{Patients characteristics}

Between December 2014 and February 2017, 18 patients with a median age of 73 years were enrolled into the TCP/ ATRA trial (Fig. 1). Three patients dropped out because they received study medication for less than 14 days. Two of them had intolerance to TCP and one had rapid AML progression and died. These patients were not evaluable for response and survival analysis.

Table 1 displays the clinicopathological characteristics of all patients. Four patients were refractory and among the 14

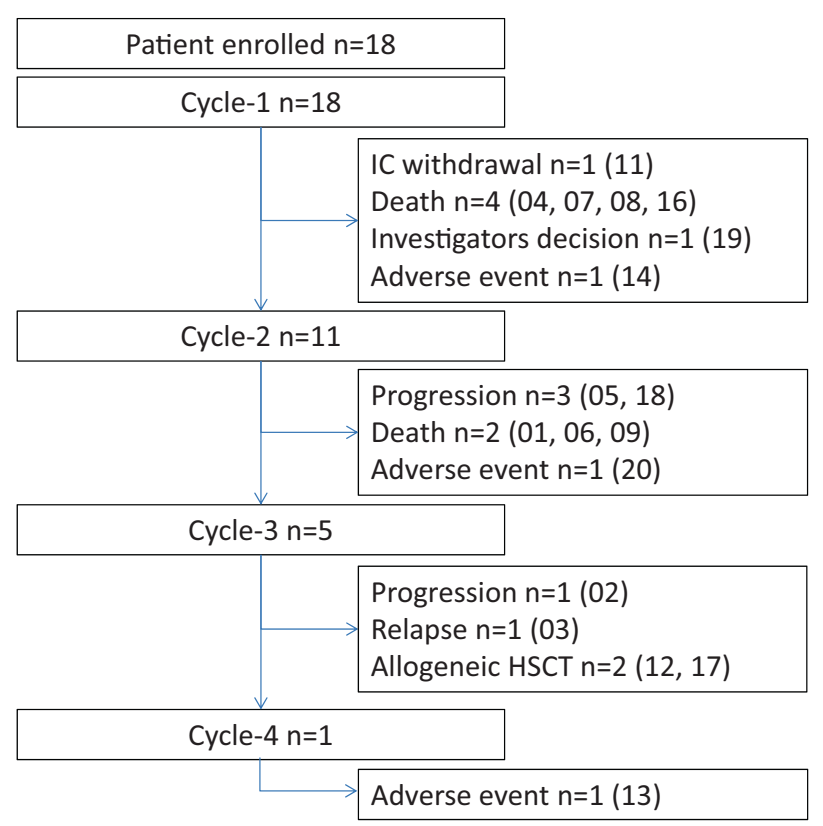

Fig. 1 Consort diagram of the TCP/ATRA trial. The clinical course of all 18 patients treated in the study is depicted. Reasons for end of treatment are indicated per cycle. Patient UPN are shown in brackets. HSCT hematopoietic stem cell transplantation, IC informed consent. 
Table 1 Patient demographics and baseline characteristics.

\begin{tabular}{|c|c|}
\hline & $N=18$ \\
\hline Median (range) age, years & $73(22-79)$ \\
\hline Male/female, $n$ & $10 / 8$ \\
\hline \multicolumn{2}{|l|}{ ECOG performance status, $n(\%)$} \\
\hline $0-1$ & $11(61.1)$ \\
\hline $2-3$ & $7(38.9)$ \\
\hline \multicolumn{2}{|l|}{ Disease status, $n(\%)$} \\
\hline Refractory & $4(22.2)$ \\
\hline Relapsed & $14(77.8)$ \\
\hline \multicolumn{2}{|l|}{ Median of previously therapy lines $(n)$} \\
\hline Refractory & $1.5(1 n=2,2 n=2)$ \\
\hline Relapsed & $2.5(1 n=5,2 n=2, \geq 3 n=7)$ \\
\hline Previously allogeneic HSCT, $n$ & 8 \\
\hline \multicolumn{2}{|l|}{ Cytogenetics, $n(\%)$} \\
\hline Normal & $3(16.7)$ \\
\hline Unfavorable & $13(72.2)$ \\
\hline Missing & $2(11.1)$ \\
\hline \multicolumn{2}{|c|}{ Baseline peripheral blood / bone marrow (range) } \\
\hline Median (range) WBC count, G/L & $3.51(1.27-24.44)$ \\
\hline $\begin{array}{l}\text { Median (range) peripheral } \\
\text { blasts, \% }\end{array}$ & $15(0-89)$ \\
\hline $\begin{array}{l}\text { Median (range) bone marrow } \\
\text { blasts, } \%\end{array}$ & $52.5(10-100)$ \\
\hline
\end{tabular}

ECOG Eastern cooperative oncology group, HSCT hematopoietic stem cell transplantation, $W B C$ white blood cells.

relapsed patients, 8 patients had relapsed after allogeneic HSCT and 9 patients had at least two or more previous therapy lines. Half of the patients had secondary AML (eight after myelodysplastic syndrome [MDS] and one with therapy-related AML) and almost two-third belonged to the poor-risk cytogenetics group according to the ELN (European LeukemiaNet) recommendations [6].

\section{Safety and tolerability}

Median duration of treatment was 1.4 cycles (range $0.5-3.5)$. The major reasons for study termination were refractory/progressive disease $(n=6)$ or infectious complications which in the assessment of the treating physician were likely due to underlying AML $(n=5)$. In three patients, the study medication was withdrawn due to intolerance (depression $\mathrm{CTC}^{\circ} \mathrm{III}, n=1$; confusion $\mathrm{CTC}^{\circ}$ II/III $n=2$ ). None of these AEs were rated as dose-limiting toxicities (DLT). A dose reduction of TCP was necessary in five patients for the following reasons: vertigo $\mathrm{CTC}^{\circ} \mathrm{III}$ ( $n=2,60 \mathrm{mg} /$ day to $40 \mathrm{mg} /$ day and $50 \mathrm{mg} /$ day to $30 \mathrm{mg} /$ day, respectively), muscle twitching $\mathrm{CTC}^{\circ}$ II $(60 \mathrm{mg} / \mathrm{day}$ to $50 \mathrm{mg} /$ day $)$, and confusion $\mathrm{CTC}^{\circ} \mathrm{II} / \mathrm{III}(n=2 ; 60 \mathrm{mg} /$ day to 30 and $20 \mathrm{mg} /$ day, respectively). In all these cases, the dose could not be increased again and the two patients with confusion were withdrawn from the study and died after termination of the study from AML progression. In one patient ATRA was paused for $48 \mathrm{~h}$ due to a differentiation syndrome. Of 158 AEs reported, 43 were considered to be drug-related ( 38 to TCP; 4 to ATRA; 1 to both) and 39 of these were non-hematological AEs (Table 2). Overall, the 43 treatment-related AEs were reported in 13 patients (72\%). The most common AEs of any grade associated with TCP treatment were hypotension, orthostatic dysregulation, vertigo, and confusion, which were mostly mild. Eleven TCP/ATRA-related grade $\geq 3$ AEs were reported in 7 patients (39\%). Irrespective of relatedness, 53 grade $\geq 3$ AEs were reported in 16 patients. The most common of which were infections, thrombocytopenia, and anemia, the latter two occurred in three patients. There were a total of 18 serious AEs (SAEs) of any causality. None of the SAEs were defined as serious adverse reaction (SARs) or suspected unexpected SARs (SUSARs). No significant clinical interaction between TCP and ATRA was observed in term of side effects. A full description of all AEs and SAEs can be found in the Supplement (Supplementary Tables S1 and S2).

\section{Treatment response and survival}

With a median follow-up of 15.5 months (95\% confidence interval (CI), 13.9-NA), 17 (94\%) of the patients have died. The clinical course of all study patients is depicted in Supplementary Fig. S1. Three out of the 15 evaluable patients had an objective response to TCP/ATRA treatment (Table 3). Two patients achieved a CRi after the first cycle. Median OS of the treated study cohort was 3.36 months (95\% CI, 1.38-NA) (Fig. 2).

Both patients who reached CRi presented at the time of inclusion with refractory disease and unfavorable cytogenetics (Supplementary Table S3). One patient with CRi was withdrawn from the study after two cycles after significant clinical status improvement to undergo allogeneic HSCT. At 20 days after the end of treatment (EOT) and before start of conditioning therapy for allogeneic HSCT the patient relapsed. The other patient achieving a CRi was a 75-year old male with secondary AML, who was refractory after intensive induction therapy with $30 \%$ blasts in the bone marrow (Fig. 3a-c). The patient was then enrolled in the TCP/ATRA trial and reached CRi after the first cycle. He continued with the second cycle, but he developed leukemic skin infiltration without evidence of blasts in the bone marrow. Therefore, the treating physicians decided to continue with a third cycle outside of this trial in addition to treatment with 5-azacitidine. At the end of cycle three, the patient had subtotal bone marrow infiltration. He died 5 months later from progressive disease. A PR was reported in one patient after the first cycle, lasting for 4 cycles. But 
Table 2 Treatment-related adverse events according to NCI CTC version 4.0 during the TCP/ATRA trial.

\begin{tabular}{|c|c|c|c|c|c|}
\hline \multirow[t]{3}{*}{ Events } & \multicolumn{5}{|c|}{ Grade } \\
\hline & & I & II & III & IV \\
\hline & $n$ & $n$ & $n$ & $n$ & $n$ \\
\hline \multicolumn{6}{|l|}{ Non-hematological } \\
\hline Vertigo & 7 & 3 & 3 & 1 & 0 \\
\hline Hypotension & 4 & 2 & 1 & 1 & 0 \\
\hline Orthostatic dysregulation/syncope & 4 & 1 & 3 & 0 & 0 \\
\hline Confusion/dizziness & 4 & 0 & 3 & 1 & 0 \\
\hline Skin reaction & 4 & 2 & 2 & 0 & 0 \\
\hline Fatigue & 3 & 0 & 2 & 1 & 0 \\
\hline Xerostomia & 2 & 1 & 1 & 0 & 0 \\
\hline Tinnitus & 2 & 1 & 1 & 0 & 0 \\
\hline Hearing impairment ${ }^{\mathrm{a}}$ & 1 & 0 & 0 & 1 & 0 \\
\hline Vision disorders & 1 & 1 & 0 & 0 & 0 \\
\hline Pain & 1 & 0 & 1 & 0 & 0 \\
\hline Coagulopathy ${ }^{\mathrm{a}}$ & 1 & 0 & 0 & 1 & 0 \\
\hline Mucositis & 1 & 0 & 0 & 1 & 0 \\
\hline Insomnia $^{\mathrm{b}}$ & 1 & 0 & 1 & 0 & 0 \\
\hline Muscle twitching & 1 & 0 & 1 & 0 & 0 \\
\hline Myalgia & 1 & 1 & 0 & 0 & 0 \\
\hline Depression $^{\mathrm{a}}$ & 1 & 0 & 0 & 1 & 0 \\
\hline Total number of AEs & 39 & & & & \\
\hline \multicolumn{6}{|l|}{ Hematological } \\
\hline Anemia & 1 & 0 & 0 & 1 & 0 \\
\hline Thrombocytopenia & 1 & 0 & 0 & 0 & 1 \\
\hline Leukocytosis $^{\mathrm{a}}$ & 1 & 0 & 1 & 0 & 0 \\
\hline Differentiation syndrome ${ }^{a}$ & 1 & 0 & 0 & 1 & 0 \\
\hline Total number of AEs & 4 & & & & \\
\hline
\end{tabular}

$\overline{A E s}$ adverse events, NCI CTC National Cancer Institute Common Toxicity Criteria.

${ }^{a}$ Related to ATRA.

${ }^{\mathrm{b}}$ Related to both.

Table 3 Treatment response.

\begin{tabular}{lc}
\hline & $n=15$ \\
\hline Best response, $n(\%)$ & \\
ORR & $3(19.9)$ \\
CR & - \\
CRi & $2(13.3)$ \\
PR & $1(6.7)$ \\
SD & $4(26.7)$ \\
NR & $5(33.3)$ \\
Median overall survival (range), months & $3.36(1.38-\mathrm{NA})$ \\
\hline
\end{tabular}

$\overline{C R}$ complete remission, $C R i \mathrm{CR}$ with incomplete recovery of neutrophils/ platelets, $N A$ not applicable, $N R$ no response, $O R R$ overall response rate, $P R$ partial response, $S D$ stable disease.

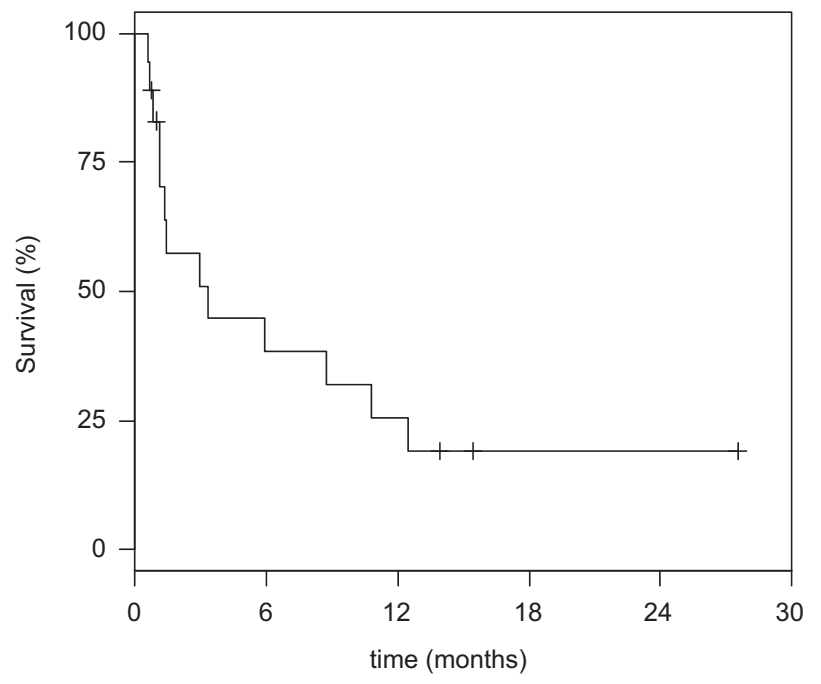

Fig. 2 Kaplan-Meier overall survival. Kaplan-Meier plots are shown for all evaluable patients.

treatment had to be stopped due to ATRA intolerance (depression $\left.\mathrm{CTC}^{\circ} \mathrm{III}\right)$. One patient had a significant reduction of bone marrow blasts (20\% to $4 \%$ ) without fulfilling PR criteria due to persisting blasts in peripheral blood. SD was reported in 4 patients. One of these patients showed a significant improvement in clinical status and underwent allogeneic HSCT from an unrelated donor after the second cycle. This patient is still alive and in clinical remission (CR with $100 \%$ donor cell chimerism) at the time of manuscript preparation. The remission status and the correlating pre- and post-treatment bone marrow blast percentages are shown in Fig. S2 (Supplementary Fig. S2).

We also observed myeloid differentiation upon TCP and ATRA treatment in patients who did not reach clinical remission. A 33-year-old male, who was refractory after two cycles of induction therapy including high dose cytarabine, underwent HSCT from his sibling donor. He was then preemptively treated with 5 -azacitidine but developed morphological relapse one year later. Being refractory to intensive salvage therapy with FLAG-IDA (Fludarabine, Cytarabine, Idarubicin, G-CSF) the patient was treated with hydroxyurea prior to study registry. Upon trial inclusion, TCP dose escalation was well tolerated and ATRA was started on day 7 without delay (Fig. 4a). On day 5 of the first cycle, hydroxyurea was stopped due to decreasing white blood cells (WBC, $1.5 \mathrm{Gpt} / \mathrm{l}$ ). After ATRA initiation, WBC rapidly increased to $53.5 \mathrm{Gpt} / 1$ within five days (Fig. 4b). At the same time, the patient developed dyspnea, fever, and hypotension. The CT-scan showed non-specific pulmonary infiltrates and pleural effusions (Fig. 4d). On day 12 we observed dominant monocytic differentiation of the blasts in peripheral blood (Fig. 4c). Since the typical signs of a differentiation syndrome were fulfilled, the patient was treated with dexamethasone and a single dose of 
Fig. 3 Clinical course of an AML patient reaching CRi in the TCP/ATRA trial. a Clinical course of the patient (03). b Numbers of leukocytes and thrombocytes in the peripheral blood and percentage of blasts in the bone marrow during treatment with TCP + ATRA. c Cell morphology of AML cells in the bone marrow at screening (left), at the end of the first cycle (middle), and at time of relapse (right). Bone marrow smears are stained with Pappenheim and shown at a magnification of $63 \times$. Combination of TCP and ATRA led to morphologically complete remission after one cycle of TCP + ATRA. After the end of cycle three, patient developed relapse.

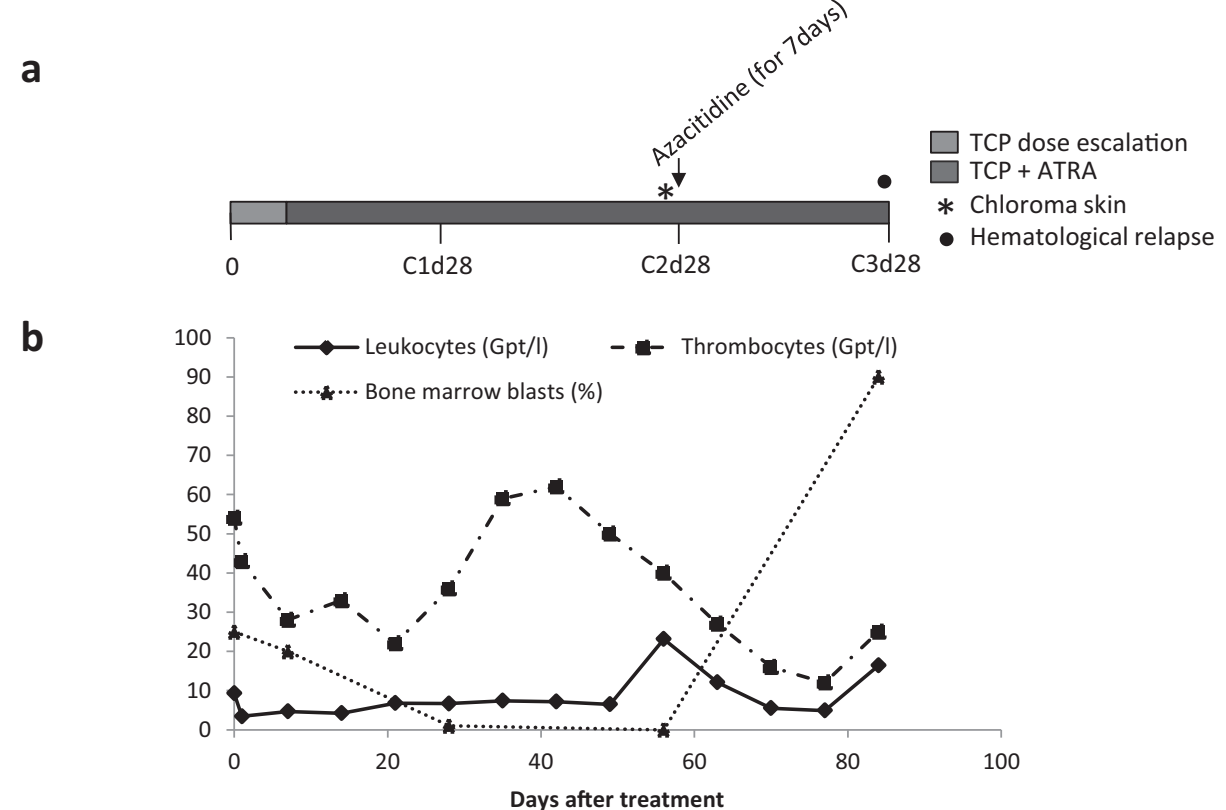

\section{C}

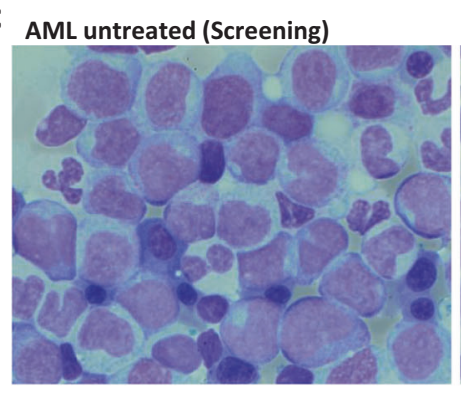

AML in CRi (C1d28)

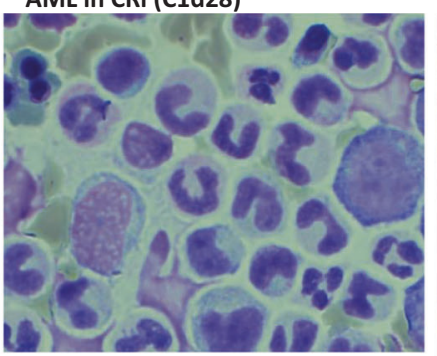

AML relapse (C3d28)

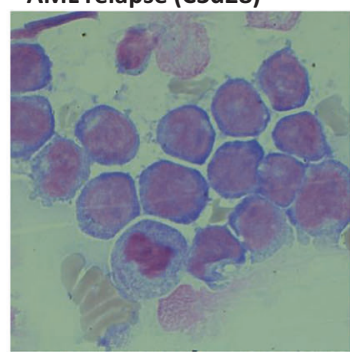

mitoxantrone ( $5 \mathrm{mg} \mathrm{IV}$ ) to alleviate the severe symptoms. ATRA was stopped for $48 \mathrm{~h}$. Subsequently, a rapid reduction of WBC $(0.5 \mathrm{Gpt} / \mathrm{l})$ was observed. The patient finished the first cycle, but the bone marrow on day 28 showed refractory disease and study participation was stopped. The patient died one month later due to AML progression.

\section{Association of TCP-levels and histone H3K4 methylation with treatment response}

TCP concentrations in the plasma of patients were analyzed at different time points: at days 1, 7, 14 and 28 of the first and second cycle, and day 28 of following cycles. TCP levels in plasma of responding patients showed a trend to higher levels than in primary progressive patients whereas patients with stable disease showed intermediate TCP levels (Fig. 5). For four study patients compatible material at different time points was available to determine intracellular TCP concentrations and global $\mathrm{H} 3 \mathrm{~K} 4$ methylation as a marker of LSD1 activity (Fig. 6a-c). All four patients had varying blast percentages in peripheral blood and bone marrow, respectively (ranging from $17-90 \%$ at screening). Sorting of cell populations was not reasonable due to limited material for the different assays after sorting. Thus, we analyzed whole-cell populations after density centrifugation. Intracellular TCP concentrations increased from screening to C1d7 and further time points during treatment (Fig. 6b), which is in line with plasma concentrations (Fig. 6a). This clearly demonstrates the intracellular uptake of TCP within our clinical trial, which is a prerequisite for LSD1 inhibition. For patients 03-004 and 01-002, we identified increased global H3K4 dimethylation at $\mathrm{C} 1 \mathrm{~d} 7$ and $\mathrm{C} 1 \mathrm{~d} 28 / \mathrm{C} 2 \mathrm{~d} 28$, respectively, as a potential marker of LSD1 inhibition. H3K4 monomethylation was similarly changed. For patient 03-004 blast percentages at diagnosis and C1d7 were 90\%, thus western blot results here indeed reflect the chromatin H3K4 methylation status in blasts. But, this patient could not receive ATRA at $\mathrm{C} 1 \mathrm{~d} 7$ and thus dropped out from study. In two patients (01-003 and 01-005) the changes in H3K4 methylation were inconclusive. For all patients analyzed by western blot LSD1 expression was detectable at screening and time points during treatment (Fig. 6c).

In conclusion, although we could demonstrate intracellular uptake of TCP in vivo, we cannot define any biomarkers for TCP/ATRA response due to limited availability of leukemic material within this study. 
Fig. 4 Myeloid differentiation syndrome in a patient in the TCP/ATRA trial. a Clinical course of the patient (05). b Numbers of leukocytes in the peripheral blood during the first cycle. c Cell morphology of AML cells in the peripheral blood on day one and day +12 of the first cycle. Peripheral blood smears are stained with Pappenheim and shown at a magnification of $63 \times$. Treatment with TCP and ATRA leads to morphological signs of differentiation of blasts into polynuclear leukocytes. d Axial CT-scans on day +12 show centrilobular nodules and ground-glass opacity in both lungs with predominantly small spotted alveolar infiltrates and discrete interstitial thickenings. Further pleural effusions on the right more than on the left.

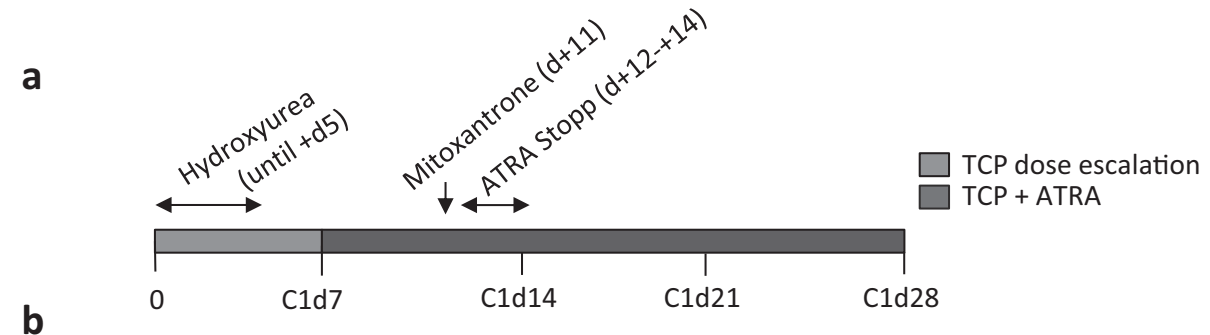

b

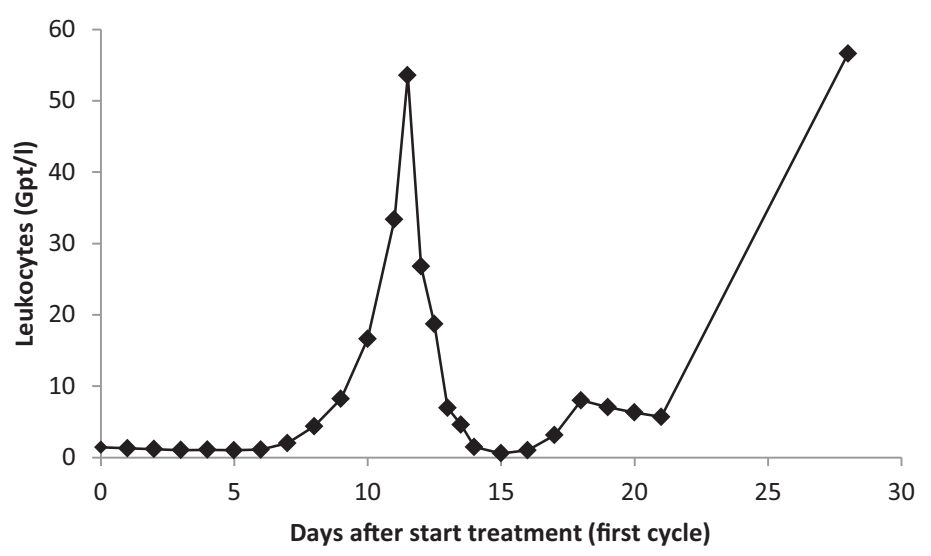

C
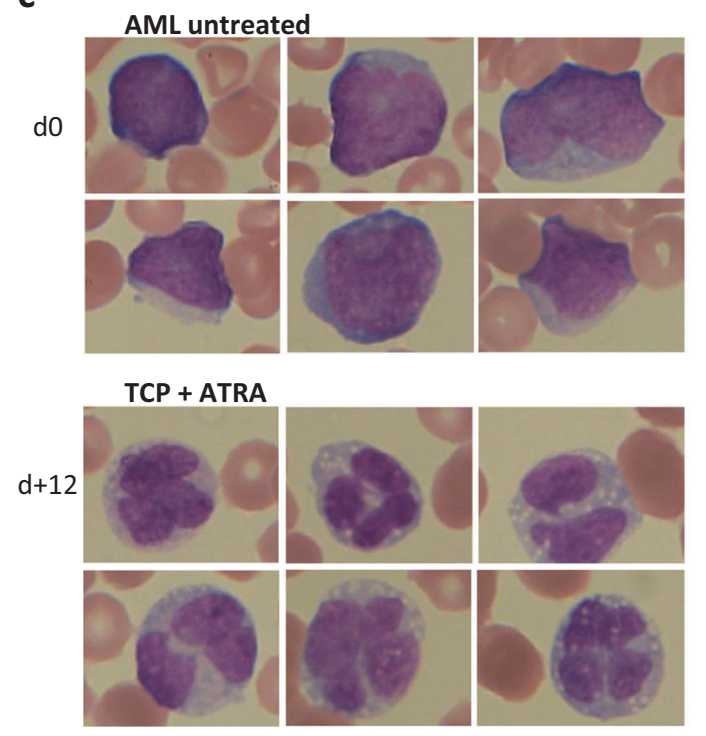

d

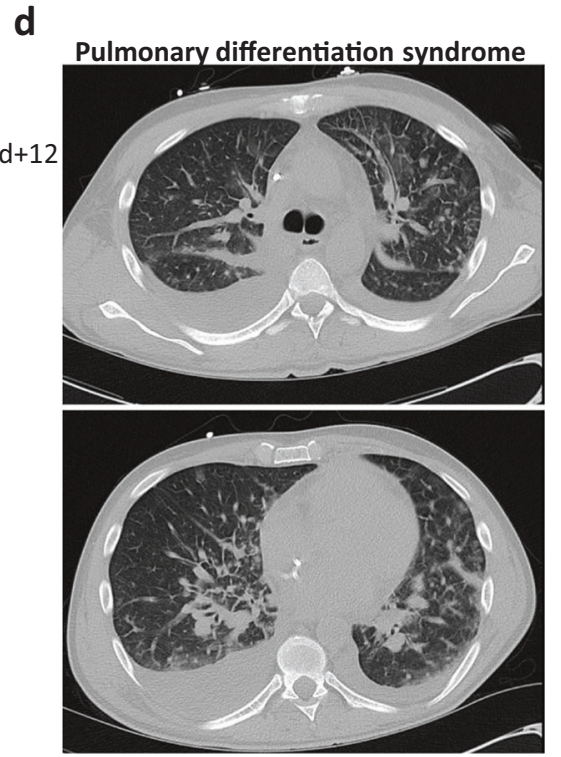

\section{Discussion}

The TCP/ATRA trial investigated the combination of ATRA and the LSD1 inhibitor TCP as salvage therapy for relapsed/ refractory ( $\mathrm{r} / \mathrm{r})$ AML patients, who were not eligible for intensive treatment. We observed an overall response rate of $20 \%$ including patients who achieved a short-lived CR with incomplete hematologic recovery. The patient cohort was highly enriched for patients with poor prognosis: $40 \%$ of patients had relapsed after allogeneic HSCT and 60\% had more than two previous therapy lines. Additionally, both patients with $\mathrm{CR}$ were refractory to cytarabine induction therapy and harbored an unfavorable karyotype.
In patients with $\mathrm{r} / \mathrm{r}$ AML, remissions are rarely achieved without intensive chemotherapy or HSCT. In patients with newly diagnosed AML, who are not intensively treatable, non-intensive treatment regimens such as low-dose cytarabine, or hypomethylating agents (HMA) alone induce CR in only $15-20 \%$ of patients and 1-year survival rates of these patient cohorts are low with 20-45\% [7-10]. The response and survival rates of patients with $\mathrm{r} / \mathrm{r}$ AML treated nonintensively are worse with a median and 2-years survival of 1.6-3.1 months and $0-4 \%$, respectively [11]. CR/CRi rates for patients treated with HMA are approximately $16 \%$ [12]. Recently, isocitrate dehydrogenase (IDH) 1 and 2 inhibitors have been shown to induce granulocytic differentiation in 


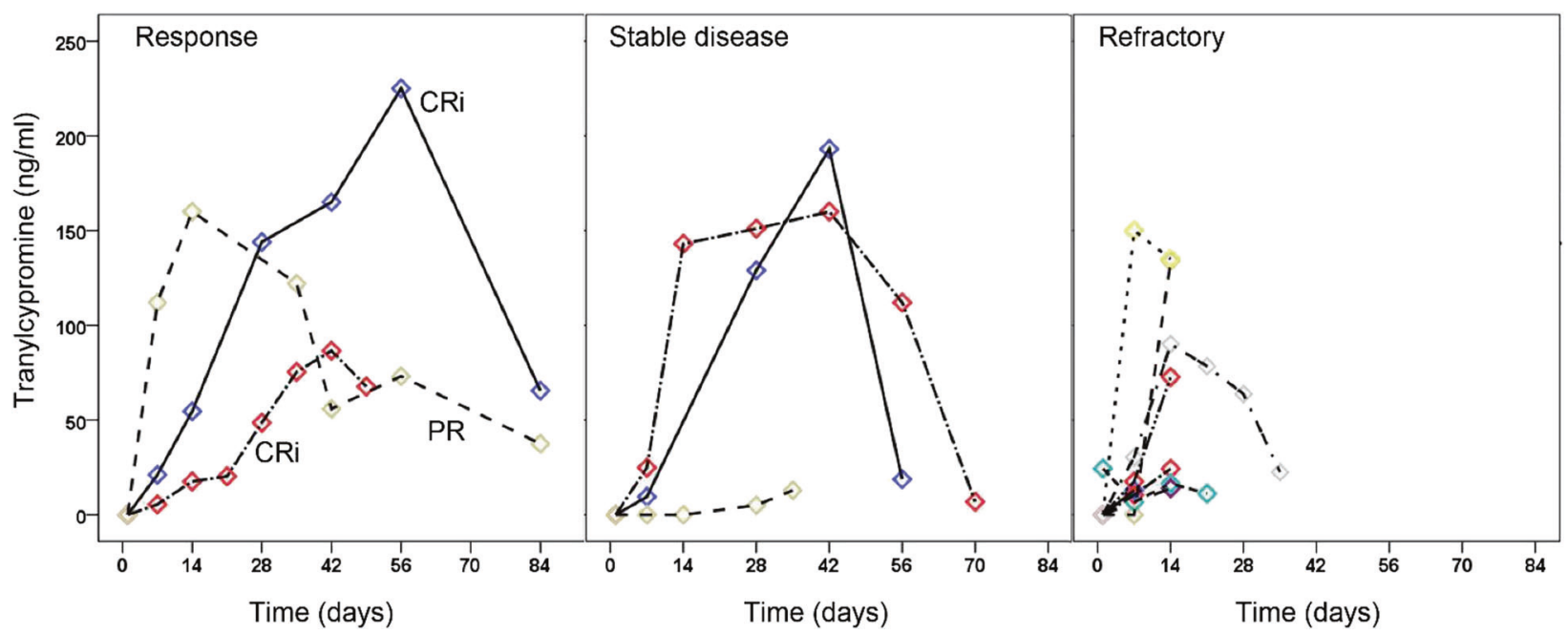

Fig. 5 Tranylcypromine levels and clinical response in the TCP/ATRA trial. The graphs depict individual patient's TCP levels over time. Patients are grouped in boxes according to clinical outcomes.

leukemic blasts and induce CR/CRi rates of $21 \%$ and $19 \%$ in $\mathrm{r} / \mathrm{r}$ AML, respectively $[13,14]$. However, these mutations are rare and occur in only $8 \%$ and $12 \%$ of all AML patients [15]. The efficacy of novel drugs such as venetoclax as single agent is limited in AML [16]. Venetoclax is much more active in combination therapy [17]. Accordingly, it is likely that combination therapies might be more successful in AML than monotherapies. This assumption demands different trial designs that incorporate strategies for synergism at an early stage of drug development.

LSD1 has recently emerged as a therapeutic target in AML with high expression in AML patients [2]. LSD inhibits differentiation, enhances proliferation, invasiveness, and cell motility in AML, and is associated with poor prognosis $[18,19]$. Knockdown of LSD1 has been shown to inhibit the growth of AML cells and induce differentiation [4, 20-22]. Different LSD1 inhibitors are currently in development [23]. We recently showed that LSD1 inhibition by TCP induced differentiation in different AML cells and primary AML patient samples [4]. This effect was enhanced when TCP was combined with ATRA via restoring transcriptional inactivated retinoid acid receptor (RAR) target genes, important for myeloid differentiation, and thus sensitizing non-APL cells to ATRA-induced differentiation [4]. The TCP/ATRA trial was designed based upon these results. The findings of myeloid differentiation and clinical responses are in line with synergistic efficacy of LSD1 inhibition and ATRA treatment in non-APL AML patients. Using mass spectrometry we demonstrated intracellular uptake of TCP into patients' blasts, a prerequisite of LSD1 inhibition. However, we were unable to define molecular markers of LSD1/ATRA response such as differences in $\mathrm{H} 3 \mathrm{~K} 4$ dimethylation of specific differentiationassociated genes detected in vitro [4] due to limited patient sample availability. We observed increases in global H3K4 methylation upon TCP application in samples of two analyzed patients. But, the use of increased global H3K4 dimethylation as a marker of LSD1 inhibition is controversially discussed in the literature and not regularly observed [4, 24, 25]. Further, varying blast levels in patients' bone marrow render it difficult to draw clear conclusions with regard to $\mathrm{H} 3 \mathrm{~K} 4$ methylation and response. Nonetheless, our data clearly indicate plasma concentrationdependent uptake into leukemic blasts in vivo. Also, the observed increases in H3K4 methylation suggest that TCP can inhibit LSD1 demethylase activity in leukemic blasts in AML patients.

TCP is a MAO inhibitor, which has been approved as an antidepressant for almost 60 years. Recently, it was the first drug to be discovered to inhibit LSD1 irreversibly [26]. After a dose-escalating phase of 7 days, we used a dosage of $60 \mathrm{mg} /$ day, which is the recommended maximum daily dose for use to treat depression according to clinical studies and summary of products characteristics [27, 28]. TCP concentrations used in the preclinical experiments are representative of the range of peak plasma concentrations reported in patients treated with this drug [4, 29]. This suggests that the dose, which is similar to the maximum dose used in depressive patients, could be an effective dose in AML patients. As expected, the most frequent TCPrelated AEs were vertigo, hypotension, orthostatic dysregulation and confusion/dizziness. In five patients, the TCP dose had to be permanently reduced down to $30 \mathrm{mg} / \mathrm{day}$ and could not be increased again. Two patients had to be withdrawn from the study due to persistent intolerance (confusion $\mathrm{CTC}^{\circ} \mathrm{II}$ and III, respectively). However, these two patients were already in a poor general condition at the time of study inclusion and died from leukemia 
a

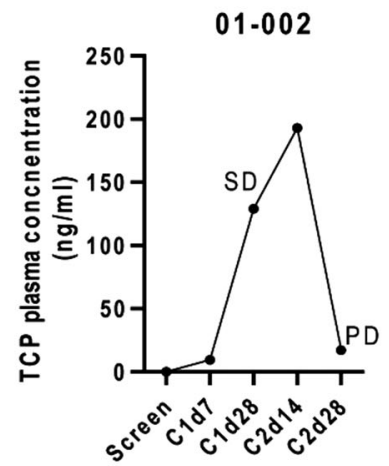

b

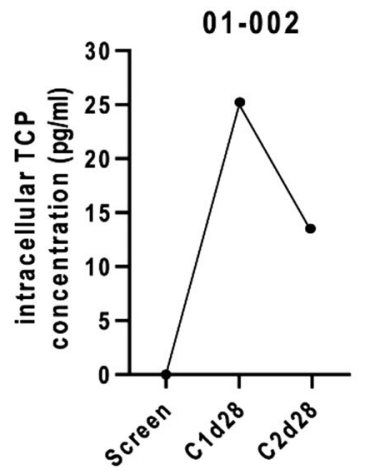

$01-003$

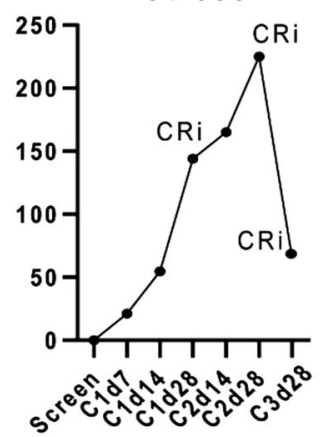

$01-003$

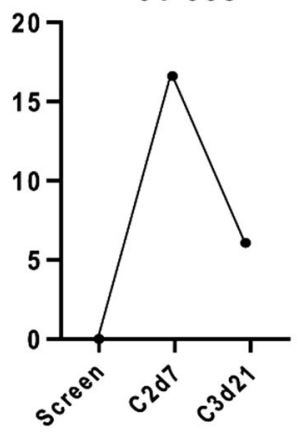

$01-005$

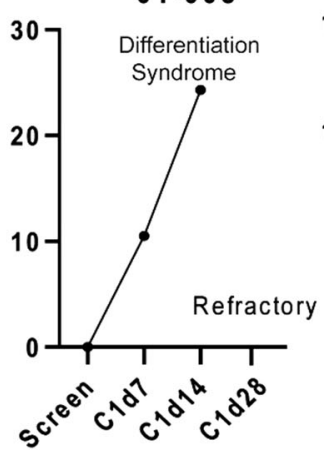

$01-005$

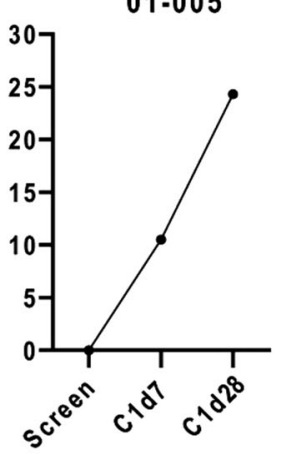

$03-004$

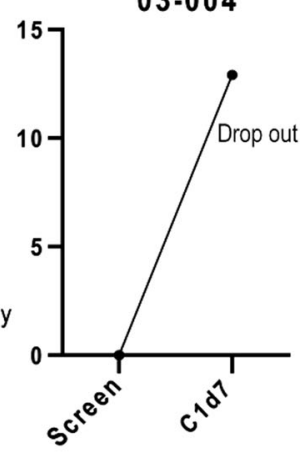

03-004

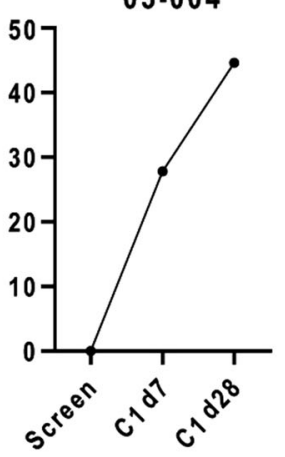

C

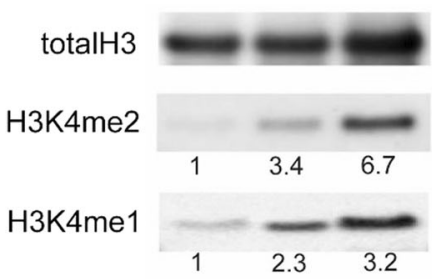

Actin

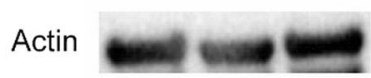

LSD1
01-003

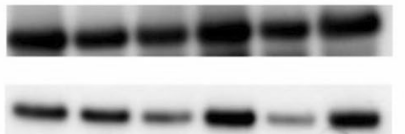

$\begin{array}{llllll}1 & 1.15 & 0.93 & 0.98 & 0.56 & 1.02\end{array}$

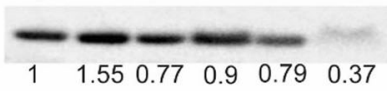

$\begin{array}{llllll}1 & 1.55 & 0.77 & 0.9 & 0.79 & 0.37\end{array}$
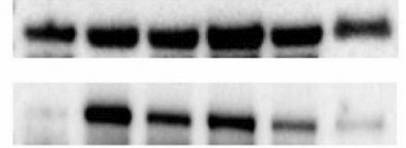

$s^{c^{e^{8^{r}}}} \mathrm{c}^{\lambda^{2^{8}}} \mathrm{c}^{2^{2^{8}}}$

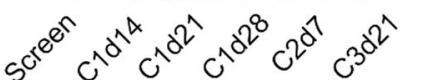

01-005

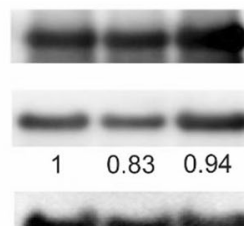

$0.97 \quad 0.6$

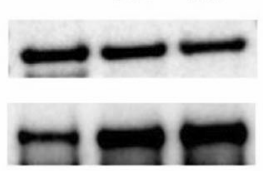

$s^{a^{e^{2}}} \mathrm{c}^{\lambda^{1}} \mathrm{c}^{d^{2^{8}}}$
03-004

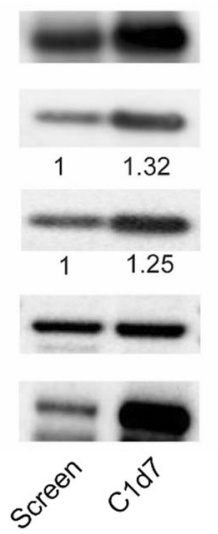

Fig. 6 Tranylcypromine plasma concentrations, intracellular concentrations and H3K4-dimethylation of selected study patients. a TCP plasma concentrations of indicated study patients at screening and during treatment course. All patients reached the maximum TCP dose of $60 \mathrm{mg} /$ day by day 7 . b Intracellular TCP concentrations of indicated study patients at screening and during treatment course. c H3K4me1, H3K4me2, totalH3, LSD1 and Actin protein expression of indicated study patients at screening and during treatment course. $\mathrm{H} 3 \mathrm{~K} 4 \mathrm{me} 1 /$ totalH3 and $\mathrm{H} 3 \mathrm{~K} 4 \mathrm{me} 2 /$ totalH3 ratios were calculated using ImageJ. Ratios at screening are depicted as a reference value of " 1 ". Ratios obtained at additional treatment time points are set in proportion accordingly. progression shortly after the end of the study medication. In addition one of these patients had concomitant infection, which might be reasonable cause for confusion, too. Thus, we did not refer this AE as DLT. Overall, the combination of TCP and ATRA was safe but most patients experienced side effects at higher doses. Different TCP-derived inhibitors, such as ORY1001 or IMG7289, with higher specificity to LSD1 than for MAO could be used to minimize TCP- related side effects. These drugs are still tested in ongoing phase I/II studies, and no safety data have been published so far [19]. Alternatively, reversible LSD inhibitors with safer metabolic profile have shown promising preclinical antileukemic properties [19]. For example, SP2509 inhibits cell proliferation and promotes cell differentiation and apoptosis in AML cell lines and primary AML cells without inducing any unrelated in vivo toxicity [30]. 
In conclusion, treatment with TCP and ATRA is a safe treatment for $\mathrm{r} / \mathrm{r}$ AML patients. LSD1 inhibition combined with ATRA induces differentiation and clinical response in AML patients. Thus, this clinical trial provides evidence for an LSD1 inhibition/ATRA treatment paradigm. Patients showing response had higher TCP levels compared to nonresponders in our trial. However, maintaining high levels may be problematic given the dose-limiting side effects. Therefore, novel LSD1 inhibitors might reach and maintain more effective levels in AML patients, while causing fewer side effects. Particularly, combination of inhibitors of histone modifiers with differentiation drugs might emerge as a powerful new approach in AML therapy.

Acknowledgements This study was supported by Deutsche Krebshilfe e.V. (110500), and by DFG MU 1328/15-1 and MU1328/9-2 (all to CMT) and by Sander Stiftung Nr. 2017.004.1 (to SG and CMT), Germany.

Author contributions Conception and design: CMT, PM, SG, MD. Collection and assembly of data: MW, PM, JG, RN, CS, J-HM, GL, MD, MW, CR, MB, HS, UP, SG, BB, KIF, JB, WEH, LPM, MB, CP, CMT. Central diagnostics: MW, PM, CMT. Statistical analysis: RS, MD, CMT. Interpretation: MW, CP, SG, RS, MD, CMT. Manuscript writing: MW, SG, CP, RS, CMT. Final approval of manuscript: All coauthors reviewed and approved the final manuscript.

\section{Compliance with ethical standards}

Conflict of interest The authors declare that they have no conflict of interest.

Publisher's note Springer Nature remains neutral with regard to jurisdictional claims in published maps and institutional affiliations.

\section{References}

1. Abdel-Wahab O, Levine RL. Recent advances in the treatment of acute myeloid leukemia. F1000 Med Rep. 2010;2:55.

2. Berglund L, Björling E, Oksvold P, Fagerberg L, Asplund A, Szigyarto CA, et al. A genecentric Human Protein Atlas for expression profiles based on antibodies. Mol Cell Proteom. 2008;7:2019-27.

3. Dart RC, editor. Medical toxicology. 3rd ed. Philadelphia: Lippincott Williams and Wilkins; 2003.

4. Schenk T, Chen WC, Göllner S, Howell L, Jin L, Hebestreit K, et al. Inhibition of the LSD1 (KDM1A) demethylase reactivates the all-trans-retinoic acid differentiation pathway in acute myeloid leukemia. Nat Med. 2012;18:605-11.

5. Cheson BD, Bennett JM, Kopecky KJ, Büchner T, Willman CL, Estey EH, et al. Revised recommendations of the International Working Group for Diagnosis, Standardization of Response Criteria, Treatment Outcomes, and Reporting Standards for Therapeutic Trials in Acute Myeloid Leukemia. J Clin Oncol. 2003;21:4642-9.

6. Döhner H, Estey EH, Amadori S, Appelbaum FR, Büchner T, Burnett AK, et al. Diagnosis and management of acute myeloid leukemia in adults: recommendations from an international expert panel, on behalf of the European LeukemiaNet. Blood. 2010;115: 453-74.
7. Burnett AK, Milligan D, Prentice AG, Goldstone AH, McMullin MF, Hills RK, et al. A comparison of low-dose cytarabine and hydroxyurea with or without all-trans retinoic acid for acute myeloid leukemia and high-risk myelodysplastic syndrome in patients not considered fit for intensive treatment. Cancer. 2007;109:1114-24.

8. Fenaux P, Mufti GJ, Hellström-Lindberg E, Santini V, Gattermann N, Germing U, et al. Azacitidine prolongs overall survival compared with conventional care regimens in elderly patients with low bone marrow blast count acute myeloid leukemia. J Clin Oncol. 2010;28:562-9.

9. Dombret H, Seymour JF, Butrym A, Wierzbowska A, Selleslag D, Jang $\mathrm{JH}$, et al. International phase 3 study of azacitidine vs conventional care regimens in older patients with newly diagnosed AML with $>30 \%$ blasts. Blood. 2015;126:291-9.

10. Kantarjian HM, Thomas XG, Dmoszynska A, Wierzbowska A, Mazur G, Mayer J, et al. Multicenter, randomized, open-label, phase III trial of decitabine versus patient choice, with physician advice, of either supportive care or low-dose cytarabine for the treatment of older patients with newly diagnosed acute myeloid leukemia. J Clin Oncol. 2012;30:2670-7.

11. Schlenk RF, Müller-Tidow C, Benner A, Kieser M. Relapsed/ refractory acute myeloid leukemia: any progress? Curr Opin Oncol. 2017;29:467-73.

12. Stahl M, DeVeaux M, Montesinos P, Itzykson R, Ritchie EK, Sekeres MA, et al. Hypomethylating agents in relapsed and refractory AML: outcomes and their predictors in a large international patient cohort. Blood Adv. 2018;2:923-32.

13. DiNardo CD, Stein EM, de Botton S, Roboz GJ, Altman JK, Mims AS, et al. Durable remissions with ivosidenib in IDH1mutated relapsed or refractory AML. $N$ Engl $J$ Med. 2018;378:2386-98.

14. Stein EM, DiNardo CD, Pollyea DA, Fathi AT, Roboz GJ, Altman $\mathrm{JK}$, et al. Enasidenib in mutant $I D H$ relapsed or refractory acute myeloid leukemia. Blood. 2017;130:722-31.

15. Papaemmanuil E, Gerstung M, Bullinger L, Gaidzik VI, Paschka P, Roberts ND, et al. Genomic classification and prognosis in acute myeloid leukemia. N Engl J Med. 2016;374: 2209-21.

16. Konopleva M, Pollyea DA, Potluri J, Chyla B, Hogdal L, Busman $\mathrm{T}$, et al. Efficacy and biological correlates of response in a phase ii study of venetoclax monotherapy in patients with acute myelogenous leukemia. Cancer Disco. 2016;6:1106-17.

17. DiNardo CD, Pratz K, Pullarkat V, Jonas BA, Arellano M, Becker $\mathrm{PS}$, et al. Venetoclax combined with decitabine or azacitidine in treatment-naive, elderly patients with acute myeloid leukemia. Blood. 2019;133:7-17.

18. Yang GJ, Lei PM, Wong SY, Ma DL, Leung CH. Pharmacological inhibition of LSD1 for cancer treatment. Molecules. 2018;23:3194.

19. Magliulo D, Bernardi R, Messina S. Lysine-specific demethylase $1 \mathrm{~A}$ as a promising target in acute myeloid leukemia. Front Oncol. 2018;8:255.

20. Fang J, Ying H, Mao T, Fang Y, Lu Y, Wang H, et al. Upregulation of CD11b and CD86 through LSD1 inhibition promotes myeloid differentiation and suppresses cell proliferation in human monocytic leukemia cells. Oncotarget. 2017;8:85085-101.

21. Harris WJ, Huang X, Lynch JT, Spencer GJ, Hitchin JR, Li Y, et al. The histone demethylase KDM1A sustains the oncogenic potential of MLL-AF9 leukemia stem cells. Cancer Cell. 2012;21:473-87.

22. McGrath JP, Williamson KE, Balasubramanian S, Odate S, Arora $\mathrm{S}$, Hatton C, et al. Pharmacological inhibition of the histone lysine demethylase KDM1A suppresses the growth of multiple acute myeloid leukemia subtypes. Cancer Res. 2016;76:1975-88. 
23. Morera L, Lübbert M, Jung M. Targeting histone methyltransferases and demethylases in clinical trials for cancer therapy. Clin Epigenetics. 2016;8:57.

24. Barth J, Abou-El-Ardat K, Dalic D, Kurrle N, Maier AM, Mohr S, et al. LSD1 inhibition by tranylcypromine derivatives interferes with GFI1-mediated repression of PU.1 target genes and induces differentiation in AML. Leukemia. 2019;33:1411-26.

25. Schulte JH, Lim S, Schramm A, Friedrichs N, Koster J, Versteeg $\mathrm{R}$, et al. Lysine-specific demethylase 1 is strongly expressed in poorly differentiated neuroblastoma: implications for therapy. Cancer Res. 2009;69:2065-71.

26. Lee MG, Wynder C, Schmidt DM, McCafferty DG, Shiekhattar R. Histone $\mathrm{H} 3$ lysine 4 demethylation is a target of nonselective antidepressive medications. Chem Biol. 2006;13:563-7.
27. Thase ME, Madhukar HT, Rush AJ. MAOIs in the contemporary treatment of depression. Neuropsychopharmacol. 1995;12: $185-219$.

28. Aristo Pharma GmbH. Fachinformation Jatrosom $10 \mathrm{mg}$. Aristo Pharma GmbH, 2010. https://www.fachinfo.de/.

29. Huang Y, Stewart TM, Wu Y, Baylin SB, Marton LJ, Perkins B, et al. Novel oligoamine analogues inhibit lysine-specific demethylase 1 and induce reexpression of epigenetically silenced genes. Clin Cancer Res. 2009;15:7217-28.

30. Fiskus W, Sharma S, Shah B, Portier BP, Devaraj SG, Liu K, et al. Highly effective combination of LSD1 (KDM1A) antagonist and pan-histone deacetylase inhibitor against human AML cells. Leukemia. 2014;28:2155-64.

\section{Affiliations}

Maxi Wass ${ }^{1} \cdot$ Stefanie Göllner ${ }^{2}$ - Birgit Besenbeck ${ }^{2} \cdot$ Richard F. Schlenk $^{2} \cdot$ Petra Mundmann ${ }^{3}$ - Joachim R. Göthert ${ }^{4}$. Richard Noppeney ${ }^{4}$ Christoph Schliemann ${ }^{5} \cdot$ Jan-Henrik Mikesch ${ }^{5} \cdot$ Georg Lenz $z^{5}$ Martin Dugas $\mathbb{1}^{5}$. Martin Wermke $^{6} \cdot$ Christoph Röllig $^{6} \cdot$ Martin Bornhäuser $^{6} \cdot$ Hubert Serve $^{7} \cdot$ Uwe Platzbecker $^{8}$.

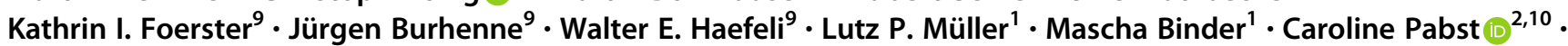
Carsten Müller-Tidow $\mathbb{1}^{2,10} \cdot$ on behalf of the Study Alliance Leukemia (SAL)

1 Department of Hematology and Oncology, University Hospital Halle, Halle (Saale), Germany

2 Department of Medicine V, Hematology, Oncology and Rheumatology, University Hospital Heidelberg, Heidelberg, Germany

3 Department of Hematology and Oncology, Paracelsus-Klinik, Osnabrück, Germany

4 Department of Hematology, University Hospital Essen, Essen, Germany

5 Department of Medicine A, University Hospital Münster, Münster, Germany
6 Medical Clinic I, University Hospital Carl Gustav Carus, Dresden, Germany

7 Department of Medicine II, Hematology/Oncology, Goethe University, Frankfurt/Main, Germany

8 Department of Medicine I, Hematology, Cellular Therapy, Hemostaseology, University of Leipzig, Leipzig, Germany

9 Department of Clinical Pharmacology and Pharmacoepidemiology, University Hospital Heidelberg, Heidelberg, Germany

10 Molecular Medicine Partnership Unit (MMPU), University of Heidelberg and European Molecular Biology Laboratory (EMBL), Heidelberg, Germany 